\title{
Probe Reaction Time Changes with Fatigue Induced by Climbing Up- and -Down Stairs
}

\author{
Qiuchen Huang ${ }^{1)}$, Hitoshi Maruyama ${ }^{1)}$, Ming Huo ${ }^{1)}$ \\ 1) Department of Physical Therapy, Faculty of Heath Science, International University of Health and \\ Welfare: 2600-1 Kitakanemaru, Ohtawara City, Tochigi 324-8501 Japan. TEL: +81 80-6799-9938, \\ E-mail: qiuchen_1984@126.com
}

\begin{abstract}
Purpose] The purpose of this study was to investigate the relationship between Probe Reaction Time (P-RT) and fatigue in normal young adults induced by climbing up and down stairs. This study examined how P-RT changes depending on the degree of fatigue. [Subjects] Twelve healthy subjects (4 males, 8 females) participated in this study. [Methods] Subjects were asked to climb up and down stairs while the P-RT and the heart rate (HR) were measured. The speed of climbing up and down stairs was decided by the subjects. [Results] The general ANOVA was significant and showed that P-RT after climbing up and down several times was different from that of the first time. Furthermore, the HR and P-RT were correlated. [Conclusion] The result of this research show that when someone is tired by climbing stairs, their P-RT is likely to be longer.

Key words: P-RT, Climbing stairs, Fatigue
\end{abstract}

(This article was submitted May 7, 2012, and was accepted May 29, 2012)

\section{INTRODUCTION}

There have been many studies of on the Probe Reaction Time (P-RT), and the P-RT has also been widely used to forecast fall risk. Past studies of P-RT have focused on walking, mental fatigue and so on. However, few have researched the relationship between P-RT and fatigue when climbing stairs.

Balance and locomotion involve multi-sensory integration while meeting environmental demands. So, when people do exercises in daily life, they must be affected by environmental factors. Reaction time is thought to influence the ability to balance ${ }^{1)}$.

Risk of falls has been assessed by using items such as vision, muscle strength, reaction time, distal sense, balance ability, and autonomic nervous system functions ${ }^{2}$. However, these items can only be measured in laboratories, and most of these measurements are difficult to use in clinical practice because of the restrictions of equipment and the environment for testing. However, P-RT and the coefficient of variation of the time of a single step are reliable and useful for evaluation of fall risk ${ }^{3)}$.

The P-RT requires a dual-task. Dual tasks are used to measure the ability to react when people carry out one task while engaged in another. Dual tasks can be used to assess a patient's performance in a functional virtual environment, and to arrange a patient's return to the community ${ }^{4}$. The P-RT measures the fastest response time in reaction to an external stimulus.

There are many previous researches of mental fatigue, and some of the studies indicat that the environment has a great affect on P-RT and that specific exercise and tired muscles also affect P-RT ${ }^{5}$. Few studies have focused on what kinds of changes occur in P-RT when a subject is tired.

Fatigue is caused by many factors, and the sense of fatigue is thought to originate in the reticular activating system of the lower brain. Musculoskeletal structures may have co-evolved with appropriate brain structures so that the complete unit functions together in a constructive and adaptive fashion ${ }^{6}$. The entire system of muscles, joints, and proprioceptive and kinesthetic functions plus parts of the brain evolved and function together in a unitary way ${ }^{7}$.

There are many evaluation methods for fatigue such as EMG, blood pressure, and heart rate (HR). Among them HR and fatigue are known to have a significant correlation. Previous research has shown that central processing by the brain is affected by fatigue. Cognitive functioning is affected by physical exhaustion ${ }^{8}$. There is also research on psychological fatigue, for example, sleep deprivation ${ }^{9)}$. Psychological state affects performance of a dual-task. Psychological and physical fatigue can be represented by HR.

A subject uses more energy climbing stairs than when walking, and this is apparent in METS. When climbing up stairs, people use 5-12 METS, but when they are walking, they use just 2-6.7 METS $^{10)}$.

The purpose of this study was to examine the relationship between P-RT and fatigue in normal young adults as they climbed up and down stairs.

\section{SUBJECTS AND METHODS}

Twelve healthy young persons with a mean age of 27.3 \pm 6.8 years, a mean height of $170.0 \pm 9.5 \mathrm{~cm}$, and a mean 
body weight of $54.6 \pm 12.3 \mathrm{~kg}$ were recruited for this study. The subjects were selected at random from among college students. All the subjects were able to independently perform activities of daily living. They gave their written consent to participation in the study after they were given an oral explanation of the procedure and objective of the study.

A pulse rate monitor (NISSEI Pulse rate monitor HR-40, Japan) was used to measure the heart rate. It was attached to the subjects' left wrist, and heart rate was measured at the left forefinger. A headphone with a microphone (ELECOM) was used to broadcast the stimulus signal to the subject. The subject's vocal response was recorded through the microphone. A digital audio player/recorder (RIO-Japan) was used to play the stimulus signals and a digital audio player/ recorder (SANYO, Japan) was used to record the vocal response that the subject made in response to the stimulus signal.

The sounds "yoi" and "pi" were pre-recorded on the RIO player. "Yoi" gave notice that the test would begin, and "pi" was the stimulus signal. The subjects were required to respond to the auditory cue "pi" by loudly saying "pa" as quickly as possible. The data were processed using DigionSound5 (Digion) software. The file was downloaded to a PC, and the time between the auditory stimulus of "pi" and the utterance of "pa", the P-RT, was evaluated.

In this research, subjects were asked to climb up and down stairs, 3 times. The speed of climbing the stairs was selfdetermined by the subjects. In the experiment a stopwatch was used to measure the time taken by subjects to climb the stairs, each time.

HR and P-RT were measured the resting in state. Then, the subject continuously climbed up and down the stairs, 3 times. While climbing the stairs, the subject was asked to respond to the auditory cues, for the evaluation of P-RT. The heart rate was measured when the subjects arrived at the first and fifth floors.

\section{RESULTS}

The data of P-RT and HR are shown in Table 1. The ANOVA shows that there were significant differences $(\mathrm{p}<0.05)$ in P-RT between: up3 and up1, up3 and down1, up3 and down2, down3 and up1; and between rest status and all 6 stair-climbing stages. Furthermore, P-RT increased with HR. The correlation coefficient between P-RT and HR was $0.63, \mathrm{p}<0.05$.

\section{DISCUSSION}

Impaired balance and mobility during the performance of concurrent cognitive or motor tasks is associated with an increased risk of adverse events, including falls, difficulty or inability to perform activities of daily living, decline in cognitive and/or physical functions, and incident disability. With a growing emphasis on evidence-based practice, rehabilitation professionals are seeking ways to translate dual-task research into clinical strategies for assessing and treating functional balance and mobility deficits in both geriatric and neurologic populations. Evidence based clinical
Table 1. Measurement of P-RT and HR on stairs

\begin{tabular}{lcc}
\hline Test & P-RT $(\mathrm{msec})$ & HR $(\mathrm{bpm})$ \\
\hline Rest status & $322.7 \pm 50.0$ & $81.7 \pm 13.0$ \\
Up 1 & $374.7 \pm 76.1$ & $135.7 \pm 16.4$ \\
Down 1 & $381.6 \pm 78.0$ & $134.9 \pm 19.4$ \\
Up 2 & $399.5 \pm 69.1$ & $147.9 \pm 18.5$ \\
Down 2 & $394.2 \pm 94.4$ & $135.9 \pm 23.0$ \\
Up 3 & $431.7 \pm 75.8$ & $153.8 \pm 18.9$ \\
Down 3 & $410.6 \pm 83.7$ & $140.6 \pm 18.1$ \\
\hline
\end{tabular}

practice requires the integration of both basic and applied research. The P-RT is an effective rehabilitation intervention for clinicians. The P-RT can be clinically used to (1) assess dual-task balance and gait, (2) improve balance and mobility under dual-task conditions, and (3) reduce the risk of adverse consequences associated with dual-task deficits.

P-RT is widely used in physical therapy as a fall risk assessment. It is greatly used in the evaluation and the treatment of senior citizens and handicapped in the clinical field.

Epidemiological studies have shown that most falls occur during locomotion. Stair negotiation is among the most challenging and hazardous type of locomotion for older people. Approximately $10 \%$ of fall-related deaths were reported to occur on stairs ${ }^{11}$.

Many falls happen suddenly, at any time during daily activities. One of major problems associated with ageing is an increased vulnerability to falls. One out of 3 elderly people is likely to fall once or more over a year. Stair negotiation is among the most challenging and hazardous types of locomotion for older people. Accidental falls may be precipitated in a situation in which seniors climb stairs while concurrently attending to unrelated tasks. When humans become elderly, their reactions to the living environment slow. The frequency of falls is even higher among elderly people in hospitals ${ }^{12)}$.

Extrapolating the results of this research, when patients or the elderly return to the community, in daily life, they should take their pulse to measure the degree of their fatigue to reduce their fall risk. It means, if a tired elderly person is on the stairs should decelerate and pay more attention to climbing up or down the stairs to reduce the risk of falling. Elderly persons should use the handrail to help maintain their balance just in case something happens.

In summary, the result of this research shows that when people feel tired on stairs, P-RT will increase, increasing the risk of falls. P-RT and HR are highly correlated, which means P-RT and the degree of fatigue are also correlated. HR and P-RT are also related to each other as shown by the energy used in climbing stairs.

\section{REFERENCES}

1) Brauer SG, Woollacott M, Shumway CA, et al.: The interacting effects of cognitive demand and recovery of postural stability in balance-impaired elderly persons. Gait Posture, 2002, 15: 83-93.

2) Lord SR, Menz HB, Tiedemann A: A physiological profile approach to falls risk assessment and prevention. Phys Ther, 2003, 83: 237-252. 
[Medline]

3) Huo M, Maruyama H: The effecttiveness of a simple approach to the assessment of the risk of falls in the elderly-P-RT and the dispersion of the time for a single step during marking time. J Phys Sci Pay, 2006, 18: $181-185$.

4) Kizony R, Fung J, Hughey L, et al.: A functional virtual environment for exploring dual task performance during locomotion. Parkinsonism Relat Disord, 2008, 14: 30. [CrossRef]

5) You YM: Simple reaction time for the impact of visual stimulation by the local fatigue dynamic muscle contraction. Jpn Soc Phys Fit Sports Med, 2003, 52: 821 .

6) Edelman GM: A biological theory of consciousness In: The remembered present. New York: Basic Books Press. 1989, pp 223.

7) Kelso JA: The self-organization of brain and behavior. In: Dynamic pat- terns. Cambridge, MIT Press. 1995, pp 115.

8) Féry YA, Hofe A: Effect of physical exhaustion on cognitive functioning. Percept Mot Skills, 1997, 84: 291-298. [Medline] [CrossRef]

9) Rogé J, Pébayle T, Hannachi S, et al.: Effect of sleep deprivation and driving duration on the useful visual field in younger and older subjects during simulator driving. Vision Res, 2003, 43: 1465-1472. [Medline] [CrossRef]

10) Waters RL, Mulroy S: The energy expenditure of normal and pathologic gait. Gait Posture, 1999, 9: 207-231. [Medline] [CrossRef]

11) Lee HJ, Chou LS: Balance control during stair negotiation in older. J Biomech, 2007, 40: 2530-2536. [Medline] [CrossRef]

12) Izumi K: In:Fall prevention based on evidence. Tokyo: Nakayama-shoten Press; 2005, p 271. 